$63^{\text {ème }}$ Congrès de la SFCO, 03007 (2015)

DOI:10.1051/sfco/20156303007

(C) Owned by the authors, published by EDP Sciences, 2015

\title{
Caractères différentiels entre la tumeur de Neumann et la tumeur d'Abrikossof : à propos de deux observations cliniques
}

\author{
Benaouf S, Khelifa M, Tabeti-Bentahar CF, Bouzouina F \\ Service de Pathologie et Chirurgie Buccales CHU Oran, Algérie
}

Introduction : La tumeur de Neumann ou tumeur gingivale à cellules granuleuses (TGCG) a été décrite pour la première fois par Neumann en 1871 [Gupta et al. 2004]. C'est une tumeur rare de la période néonatale, avec prédominance féminine dont le siège préférentiel est la crête alvéolaire supérieure [Dhingra et al. 2010]. La tumeur à cellules granuleuses (TCG) ou Tumeur d'Abrikossoff est une tumeur bénigne d'aspect nodulaire décrite pour la première fois au niveau de la langue par Abrikossoff en 1926.

Observation : Les cas rapportés sont ceux de deux nouveaux nés de sexe féminin. La première malade présentait une masse obstructive bilobée occupant la moitié de la crête alvéolaire supérieure. La patiente a été opérée sous anesthésie générale. L'exérèse n’a intéressé que le lobe antérieur de la formation, le second lobe était difficile d'accès. Les suites opératoires ont été satisfaisantes. La malade a été revue après une année, une régression spontanée et totale de la formation résiduelle a été constatée. La deuxième malade a été adressée par son pédiatre pour une formation muqueuse pédiculée de $1,5 \mathrm{~cm}$ de diamètre siégeant sur la crête alvéolaire inférieure antérieure de couleur rose. La tumeur a été excisée sous anesthésie locale. Les suites opératoires étaient satisfaisantes : aucune récidive n'a été constatée, 6 mois après le traitement chirurgical. Le résultat anatomopathologique pour les deux malades est revenu en faveur de TGCG avec de larges cellules rondes ou polygonales centrées par des noyaux ronds ou ovalaires, avec un cytoplasme granulaire.

Discussion : La TGCG et la TCG ont des caractéristiques communes dont l'aspect clinique (lésion ferme, non douloureuse et non hémorragique) et histologique (présence de cellules renfermant en intracellulaire des granules éosinophiles) [Charrier et al. 2003]. La TCG se distingue de la TGCG par sa localisation ubiquitaire : poumons, peau, colon, yeux, voies biliaires, organes sexuels... Cependant, la localisation cervico-faciale est préférentielle. Le siège lingual représente 23 à $28 \%$ des TCG et 65 à $85 \%$ des TCG de la cavité buccale [Nelson et al. 1999]. Quant à la TGCG, son siège est exclusivement gingival. L’âge des patients est aussi un signe distinctif : la TGCG est congénitale alors que la TCG touche tous les âges. Contrairement à la TCG, la régression spontanée de la TGCG est rapportée justifiant l'abstention thérapeutique dans certains cas, en l'absence de signes obstructifs [Kadlub et al. 2011]. Pour les autres, l'excision sous anesthésie locale ou générale est requise. La présence d'un réseau capillaire plexiforme et d'une hyperplasie pseudoépithéliomateuse signent la TCG. L'origine nerveuse de la TCG est admise alors qu'elle demeure controversée pour la TGCG. En effet, en immunohistochimie, la TCG est positive à la protéïne S100 et à la neuron-specific enolase alors que la TGCG est PAS positive [Nouri et al. 2008].

This is an Open Access article distributed under the terms of the Creative Commons Attribution License 4.0, which permits unrestricted use, distribution, and reproduction in any medium, provided the original work is properly cited. 
$63^{\text {ème }}$ Congrès de la SFCO

Conclusion : la TGCG et la TCG partagent des caractéristiques cliniques et histologiques nombreuses, elles ne doivent pas être confondues car la thérapeutique et le pronostic sont différents imposant le recours à l'immunohistochimie.

Nom et adresse du conférencier

Souad BENAOUF

Faculté de médecine d'Oran

114, coopérative Marhaba ES Senia Oran

31100 Oran (Algérie)

s.benaouf@yahoo.fr 